\title{
CONTROL OF OVERHEAD CRANE BY FUZZY- PID WITH GENETIC OPTIMISATION
}

\author{
A. Soukkoul, A. Khellaf2, S. Leulmil \\ 1Department of Electrotechnics, University of Skikda 21000, Algeria. 2Department of \\ electronics, University Ferhat Abbas-Sétif 19000, Algeria soukkou_a@yahoo.fr; \\ khellaf_a@yahoo.fr; leulmi_salah@yahoo.fr
}

\begin{abstract}
A fuzzy logic controller with the fuzzy knowledge base: scaling factors of the input/output variables, membership functions and the rules are optimized by the use of the genetic algorithms, is presented in this work, and its application in the highly nonlinear systems. The fuzzy structure is specified by a combination of the mixed Sugeno's and Mamdani's fuzzy reasoning. The mixed, binary-integer, coding is utilized to construct the chromosomes, which define the set of necessary prevailing parameters for the conception of the desired controller. This new controller stands out by a non standard gain (output scaling factor) which varies linearly with the fuzzy inputs. Under certain conditions, it becomes similar to the conventional PID controller with non-linearly variable coefficients. The results of simulation show, well, the efficiency of the proposed controller.
\end{abstract}

Key words: Genetic algorithms, fuzzy controller, PID

\section{INTRODUCTION}

The control of complex systems, that are nonlinear or contains an unknown dynamics, is a difficult task. In addition, the desired performances become more and more severe. It is one of the raisons that lead to the apparition of new control methods that are more sophisticated.

The apparition of the fuzzy logic formalism [1] gave birth of a new approach, where the control laws are replaced by a set of linguistic rule, determines the control strategies and objectives. This approach requires the knowledge of the human appraisal (experience). The fuzzy systems 
performances depend on the exactness of the obtained knowledge's. These problems have lead to the development of hybrid systems that could unite the training capacities of the neural networks and the decision capacities of fuzzy systems. The main objective of hybrid systems is the design of fuzzy controllers with optimum parameters.

In this hybridization, the network topology is specified by the type of the inference and by the fuzzy rules used (Sugeno's or Mamdani reasoning).

The training allows us to adjust the weights of the network, that constitute the base of the fuzzy knowledge, by the minimization of a given cost function that depends on the performances and the desired control strategy.

The backpropagation, based upon the usual gradient method, suffers from convergence problems. This constitutes a constraint that degrades the efficiency degrees of the fuzzy neural controllers.

The genetic algorithms (GA), inspired from the natural genetics [2], become a powerful competitor in the parameter optimisation domain necessary for the design of optimal fuzzy controllers.

Designing an optimal fuzzy controller is, then, a selection of an optimal vector of parameters or variables that will minimize a certain cost function subject to some constraints. Then, we can expect that simultaneous use of the combined fuzzy-genetic approaches lead to the construction of a fuzzy inference system whose structural parameters (its fuzzy knowledge base) are optimized by the GA. The main objective is to exploit the effectiveness of each to build more flexible and efficient controllers.

This article is divided in two main parts:

Description of the methodology of conception of the fuzzy neural controller.

Simulation and results of the application of the new controller on an example of nonlinear systems.

\section{METHODOLOGY OF CONCEPTION}

Mamdani's and Sugeno's models are the basis of the majority of the fuzzy controllers used in the literature of the domain of the fuzzy logic control. The main difference between the two reasonings resides in the way of assessment of the consequences of the fuzzy rules. In the method of Mamdani [3-4], every rule is represented by a conjunction (MIN) and the aggregation of the rules by a disjunction (MAX). In this model, the consequences are independent of the controller's entries. The link between the conditions and the actions is achieved, solely, by operators. 
Takagi-Sugeno and Kang [5] proposed a model to develop a systematic approach for a generation of fuzzy rules from the data set of input-output (I/O). A rule of this type has the following shape:

$$
\begin{gathered}
\text { Ri: IF ( } \left.x_{1} \text { is } A_{l 1}\right) A N D \ldots A N D\left(x_{m} \text { is } A_{m}\right) \text { THEN } \\
Y_{1}=F_{l}\left(x_{1}, x_{2}, \ldots, x_{m}\right)
\end{gathered}
$$

Generally $F_{1}(-)$ possesses a polynomial form of $x_{l}, x_{2}, \ldots$ and $x_{m}$.

$$
F_{i}\left(x_{1}, \ldots, x_{m}\right)=a_{0 i}+a_{1 i} x_{l}+\ldots+a_{m i} x_{m}
$$

This form is called the model of Sugeno of order one. In some cases, $F_{(}(-)$ must be constant (Sugeno of order zero). The determination of the coefficients of the consequences must be made by a parametric identification method [6].

The methods of defuzzification adapted to this form of rules are based on the approach: Defuzzify Without Combining $(D W C)$. The more used is the one weighted-average defuzzification, given by:

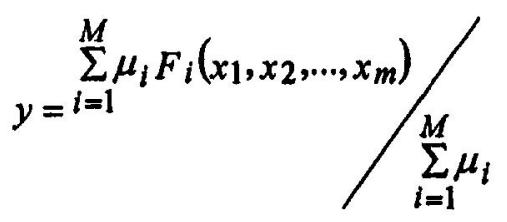

Where $\mathrm{M}$ is the number of the rules and $\boldsymbol{\mu} \mathbf{i}$ is the degree of activation of the ith rule calculated either by the operator of conjunction (MIN) or the arithmetic product.

$$
\begin{aligned}
& \mu_{i}=\mu_{i}\left(x_{1}\right) \times \mu_{i}\left(x_{2}\right) \times \mu_{i}\left(x_{3}\right) \ldots \times \mu_{i}\left(x_{m}\right) \\
& \mu_{i}=\operatorname{MIN}\left(\mu_{i}\left(x_{1}\right), \mu_{i}\left(x_{2}\right), \mu_{i}\left(x_{3}\right), \ldots, \mu_{i}\left(x_{m}\right)\right)
\end{aligned}
$$

The simplicity and the reduced execution time present two advantages of the Sugeno's approach. Its simplicity of implementation has attractes the attention of several researchers [7-8]. The main goal is to develop new technics to reduce the number of parameters of the consequences that burdens the Sugeno's method.

Hao Ying [9] proposed an analytical method of treatment of the Sugeno controller's. The output polynomials are proportional one of the other. While introducing some modifications to the level of the form of the rules, one gets: 


$$
\begin{gathered}
R_{1}: I F\left(x_{l} \text { is } A_{l l}\right) A N D \ldots A N D\left(x_{m} \text { is } A_{l m}\right) \text { THEN } \\
f_{l}=R_{l} f\left(x_{l}, \ldots, x_{m}\right) \\
R_{2}: I F\left(x_{l} \text { is } A_{2 l}\right) A N D \ldots A N D\left(x_{m} \text { is } A_{2 m}\right) \text { THEN } \\
f_{2}=R_{2} f\left(x_{l}, \ldots, x_{m}\right) \\
\vdots \\
R_{M}: I F\left(x_{1} \text { is } A_{M I}\right) A N D \ldots A N D\left(x_{m} \text { is } A_{M m}\right) \text { THEN } \\
f_{M}=R_{M} f\left(x_{l}, \ldots, x_{m}\right)
\end{gathered}
$$

Where,

$$
f\left(x_{1}, \ldots, x_{m}\right)=a_{0}+a_{1} x_{1}+\ldots+a_{m} x_{m}
$$

Using the weighted-average defuzzification method, the digital output for a set of entries $x_{1}, x_{2}, \ldots$ and $x_{m}$ is given by:

$$
U=\frac{\sum_{i=1}^{M} \mu_{i} f_{i}}{\sum_{i=1}^{M} \mu_{i}}=\frac{\sum_{i=1}^{M} \mu_{i} R_{i}}{\sum_{i=1}^{M} \mu_{i}} \cdot f\left(x_{1}, \ldots, x_{m}\right)
$$

Where $\mathrm{M}$ is the number of the rules and $\mu_{i}$ is the degree of activation of the $\mathbf{i}^{\text {th }}$ rule calculated either by the operator of conjunction (MIN) or the arithmetic product, $R_{\boldsymbol{l}}$ represents the center of gravity of the fuzzy set output (singleton) of the $\mathbf{i}^{\text {th }}$ rule.

Under the vectorial form:

$$
\vec{R}=\left(R_{1}, R_{2}, R_{3}, \ldots, R_{M}\right) ; \vec{\mu}=\left(\mu_{1}, \mu_{2}, \mu_{3}, \ldots, \mu_{n}\right) ; \vec{x}=\left(x_{1}, x_{2}, x_{3}, \ldots, x_{n}\right)
$$

The expression (7) becomes:

$$
U=G(\vec{x}, \vec{\mu}, \vec{R}) f(\vec{x})
$$

Where,

$$
G(\vec{x}, \vec{\mu}, \vec{R})=\frac{\sum_{i=1}^{M} \mu_{i} R_{i}}{\sum_{i=1}^{M} \mu_{i}}
$$


The expression $a_{0}+a_{l} x_{l}+\ldots+a_{m} x_{m}$ represents an association of the linear controllers, which contain the linear PID controller as a special case $\left(a_{0}=0\right)$. The following three-input variables are used by the discrete-time PID control in incremental form:

$$
x_{1}(n)=y_{r}(n)-y(n) ; x_{2}(n)=x_{1}(n)-x_{1}(n-1) ; x_{3}(n)=x_{2}(n)-x_{2}(n-1)
$$

Where $\boldsymbol{y}_{r}(\boldsymbol{n})$ and $y(n)$ are a set-point/reference signal for process output and the process output at sampling time $n$, respectively.

According to data of the problem to treat, the output variable can be the action of control or its variation. It is sometimes discriminating to formulate the rules of inference so that the response signal intervenes like increment of the control signal between the instants $n$ and $n-1$ :

$$
U(n)=U(n-1)+\Delta U(n)
$$

With respects of the saturation regions:

$$
U_{\min } \leq U(n) \leq U_{\max }
$$

The increment permits to introduce an integral behaviour to the global controller level. In the rest of this paper, we denote the output and the change in output as $U(n)$ and $\Delta U(n)$ at time $n$, respectively.

In the case of the direct action control, the controller's output form will be given by:

$$
\begin{aligned}
U(n) & =G(\vec{x}, \vec{\mu}, \vec{R}) f(\vec{x})=G(-)_{\left.K O P x_{1}(n)+K O D x_{2}(n)\right)} \\
& =\left(G(-)_{K O P}\right)_{x_{1}}(n)+\left(G(-)_{K O D}\right)_{x_{2}}(n)
\end{aligned}
$$

The law of PD controller in its incremental or discreet form is given by:

$$
U_{P D}(n)=\overline{K_{P}} x_{1}(n)+\overline{K_{D}} x_{2}(n)
$$

Where, $\overline{K P}$ and $\overline{K D}$ are constant gains named proportional-gain, and derivative-gain, respectively. It returns the controller equivalent to a PD with gains $\overline{K P}$ and $\overline{K D}$ variables and non-linear, since the output of a fuzzy system is non-linear in relation to these entries.

$$
\overline{K_{P}} \rightarrow G(-)_{K O P} ; \overline{K_{D}} \rightarrow G(-)_{K O D}
$$

In the case of the control variation, the output controller's will be: 


$$
\Delta U(n)=G(-)\left(a_{1} x_{1}(n)+a_{2} x_{2}(n)+a_{3} x_{3}(n)\right)
$$

By analogy with a PI and a PID in the discrete-time form, the control variation is:

$$
\begin{aligned}
& \Delta U_{P I}(n)=\overline{K_{P}} x_{2}(n)+\overline{K_{I}} x_{1}(n) \\
& \Delta U_{P I D}(n)=\overline{K_{P}} x_{2}(n)+\overline{K_{l}} x_{1}(n)+\overline{K_{D}} x_{3}(n)
\end{aligned}
$$

The relation (14) can write itself as follows:

$$
\Delta U(n)=\left(a_{1} G(-)\right) x_{1}(n)+\left(a_{2} G(-)\right)_{x_{2}}(n)+\left(a_{3} G(-)\right) x_{3}(n)
$$

This last_is similar to the one of the expression (16), only the constant gains $\overline{K_{P}}, \overline{K_{I}}$ and $\overline{K_{D}}$ are replaced by nonlinear factors: $\left(a_{2}, G(-)\right),\left(a_{1}, G(-)\right)$ et $\left(a_{3 .} G(-)\right)$, respectively.

Generally, the control of the complex systems by a PID presents a delicate task. The solution of the problem comes back to choose or to develop an efficient method for the determination of the gains of different actions, or the combination of the PID with other more sophisticated methods very adapted to the complexity and to the nature of the industrial processes.

The controller so developed presents a way of this hybridization, whose goal is to take advantage on the one hand of the precision of the mathematical model of the PID, on the other hand, of the suppleness of the elements manipulated by the fuzzy logic. What drives to the realization of a fuzzy controller to one degree of autonomy.

\section{CONTROL STRUCTURE}

Figure 1 shows the structure of the command system that contains four blocks:

- Optimization block characterized by GA.

- Structural block representing the fuzzy controller.

- Decisions block defining the performances criteria.

- System block to be controlled. 


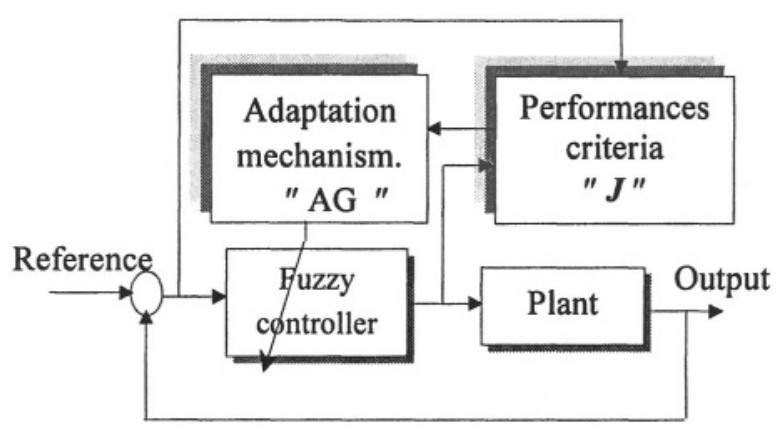

Figure 1. Control structure.

The interaction between these four blocks is summarized by the flow chart represented by figure 2 .

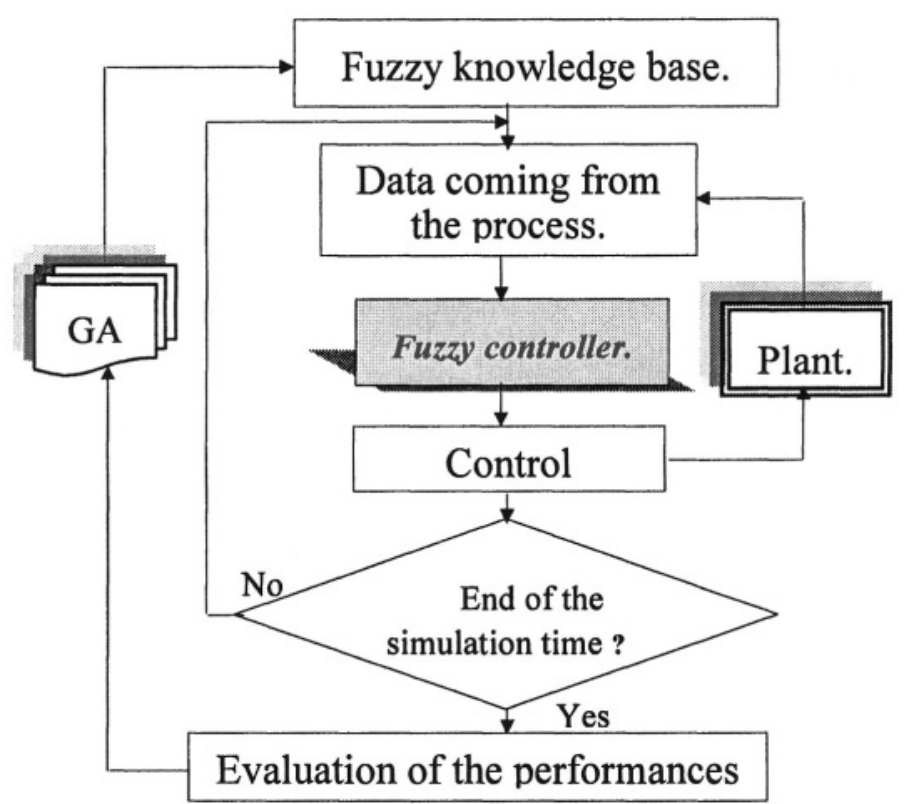

Figure 2. Interaction between the different blocks of the control structure. 


\section{PARAMETERS CODING}

The difference between the genetic algorithms used in the literature resides to the levels of the coding (binary, integer or real) and the genetic operators adapted to every coding type [13].

The introduction of the coding mixed, binary-integer, multiparametered and concatenated permits to construct the chromosome of the GA. This technique allows or encourages the coding and the successive juxtaposition of the different parameters. The parameters to optimize are:

- The widths of the fuzzy subsets associated to the spaces of $\mathrm{I} / \mathrm{O}$, binary-coded.

- The rules or the centers of the fuzzy subsets of the consequences are coded in integer. Every center is coded by a number included between 1 and the maximal number $\mathrm{N}$ of the fuzzy subsets of the output space [10-12].

- The coefficients $K_{X l}, K_{X 2}, K_{O P}, K_{O I}$ and $K_{O D}$, associated to the scaling factors of $\mathrm{I} / \mathrm{O}$ are binary-coded.

Every chromosome can represent a solution of the problem, that is, a fuzzy optimal knowledge base. Its structure is represented by the figure 3 . $L_{Z E X 1}, L_{Z E X Z}$ and $L_{Z E U}$ are the codes of the widths of the universes of discourses of the fuzzy subsets (ZE) of the entries $x_{1}, x_{2}$ and of the output $U$, respectively.

The $R_{l}$ are the codes of the conclusions characterizing the centers of the output fuzzy subsets and $K_{X 1}, K_{X 2}, K_{O P}$ and $K_{O D}$ are the codes of the coefficients of the scaling factors of the I/O variables.

\section{MECHANISM OF THE GA}

The genetic algorithms are stochastic procedures of research and exploration, inspired from the natural genetics. The figure 4 summarizes the principle of the standard GA.

The GA uses the selection by wheel of lotteries for the reproduction, two points crossover and the mutation operators. 


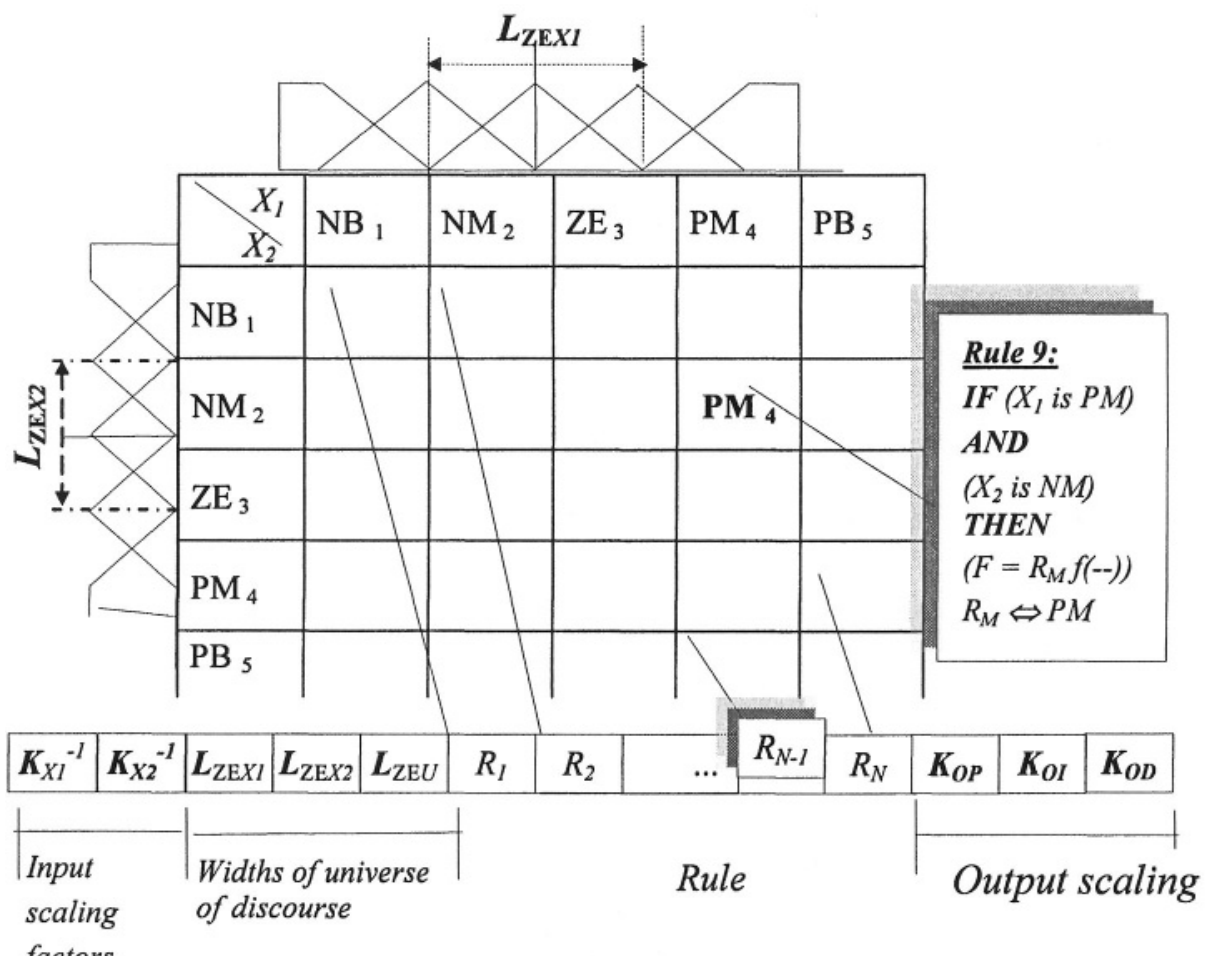

factors

Figure 3. Structure of the chromosome.

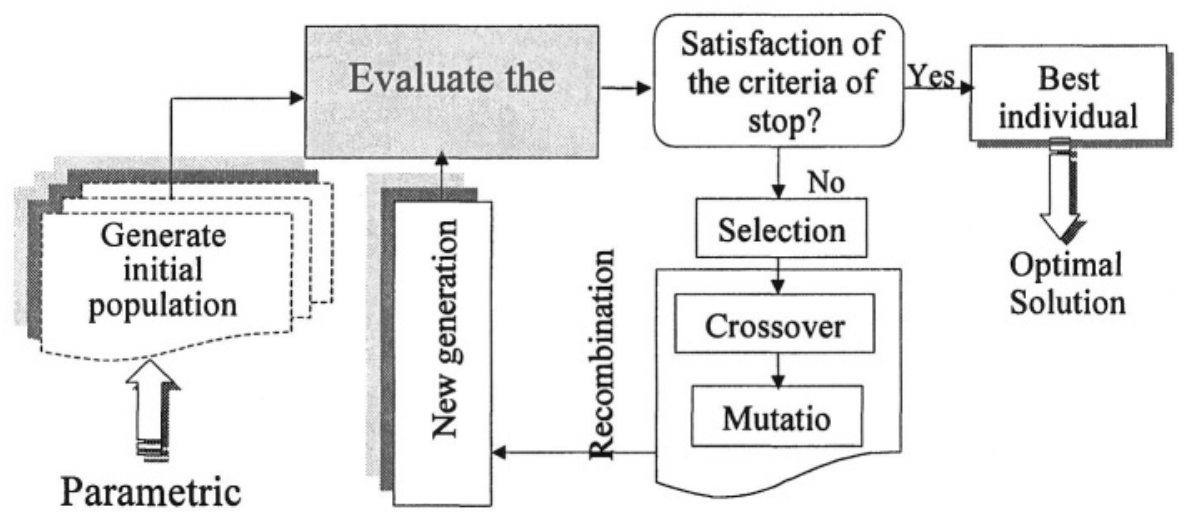

Figure 4. Execution process of the GA. 
If the mutation, takes place in the binary-coded part, the operator of mutation has the same principle that the one of the standard mutation. If the mutation takes place in the integer part, instead of changing a 1 in 0 or 0 in 1 , we change the allele by a random from value 1 to $\mathrm{N}$ different from its actual value

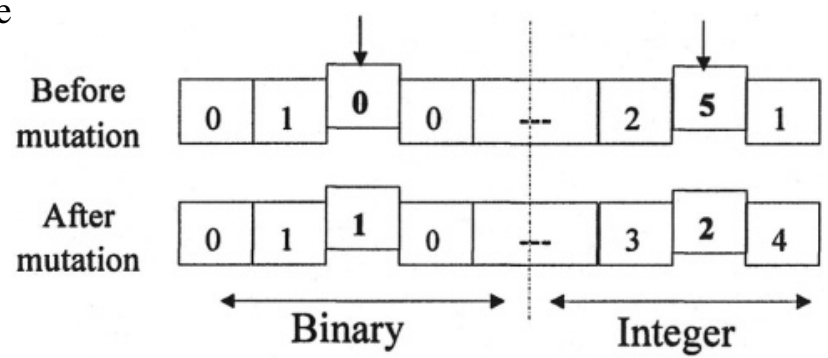

Figure 5. Mutation.

Finally, to put a limitation to the genetic divergence, one of the elitism strategies has been introduced. This last, based on the technic of the steadystate selection, permits to construct a new more effective generation than the previous. The criterion of stop of the GA is fixed by a, well determined, number of generations (Max-Gen).

The validity of the methodology and that of the form of command (PD non linear) are tested on the overhead crane considered as a non-linear, single input / multi-output system (SIMO).

\section{SIMULATION}

Figure 6 shows the schematic diagram of the crane system [14], as well as its structure. The dynamics of the system are characterized by:

$$
\begin{gathered}
\left(m_{1}+m_{2}\right) \ddot{x}+m_{2} L \ddot{\theta} \cos (\theta)-m_{2} L \dot{\theta}^{2} \sin (\theta)=F \\
m_{2} L^{2} \ddot{\theta}+m_{2} L \ddot{x} \cos (\theta)+m_{2} g L \sin (\theta)=0
\end{gathered}
$$

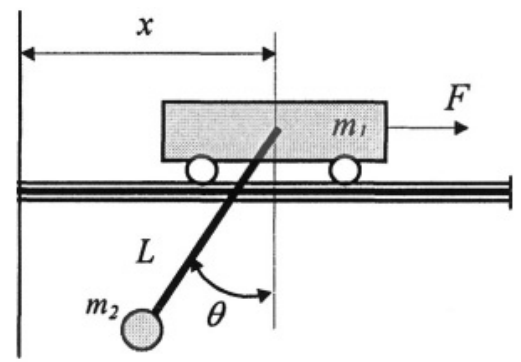

Figure 6. Overhead crane structure. 
Where, $m_{l}, m_{2}, x, L, \theta$ and $\mathrm{F}$ are the mass of the wagon, load mass, crane position, the angle of the segment in respect to the vertical axis, and driving force, respectively. The numerical used data in the simulation are: $L=1 \mathrm{~m}$, $m_{l}=1 \mathrm{~kg}, m_{2}=1 \mathrm{~kg}, g=9.8 \mathrm{~m} / \mathrm{s}^{2}$.

The objective of the control is to generate a sequences of actions $F$ (in Newton) permitting to consolidate the system in a desired state from a predetermined initial state.

The overhead crane system has four state variables $(x, \dot{x}, \theta, \dot{\theta})$. If the fuzzy controller has $N$ inputs, there will be $M^{N}$ items of rules even if every variable has $M$ fuzzy subsets. It becomes difficult to design the fuzzy controller. The solution is to divide the system in sub-systems.

Liu Diantong in [14] proposed one controller with two fuzzy subcontrollers: displacement fuzzy controller is used to control load displacement and anti-swing fuzzy controller is used to damp load swing. Figure 7 is the schematic diagram of the control system.

The adapted chromosome structure for this system has the same form of the figure 3, but doubled. A part for $\mathbf{F L C}_{\mathbf{1}}$ and the other for $\mathbf{F L C}_{2}$. The control system will be the superposition of the two outlays of the two controllers.

In this application the objective function responsible for the ordering of the chromosomes in the population is:

$$
F_{i t}=\left(1+\sum_{k=1}^{500}\left(10^{-4}\left|x_{e r r}(k)\right|+10^{-4}\left|\theta_{e r r}(k)\right|+10^{-5}|F(k)|\right)\right)^{-1}
$$

The controller thus developed $\left(\mathbf{F L C}_{1}, \mathbf{F L C}_{\mathbf{2}}\right.$ ) for this application is characterized by: $(5 \times 5 \times 5,5 \times 5 \times 5)$, i.e., five fuzzy subsets for each I/O variables. What gives to the total $25 \times 2$ rules.

Every chain of the population will contain $95 \times 2$ alleles (10 bits for each of the widths of the universe of discourses, $25 \times 2$ integers for the rules and 10 bits for every coefficient of the gains). The parameters of the GA are presented in table 1 .

Tab 1. Parameters of the GA.

\begin{tabular}{l|c}
\multicolumn{1}{c|}{ Parameter } & Value \\
\hline & \\
The size of the population. & 40 \\
The probability of mutation. & 0.03 \\
The crossover probability. & 0.8 \\
The maximal number of generation. & 500
\end{tabular}


- The parameters, $K_{X 1}$ and $K_{X 2} \in[5,40], K_{O P}$ and $K_{O D} \in[1,500]$. The initial conditions used in this simulation are: $(x, \dot{x}, \theta, \dot{\theta})=\left(10 \mathrm{~m}, 0 \mathrm{~m} / \mathrm{sec}, 30^{\circ}, 0^{0} / \mathrm{sec}\right)$.

Figure 8 indicates the evolution of the objective function. The rule base gotten at the end of execution of the GA is:
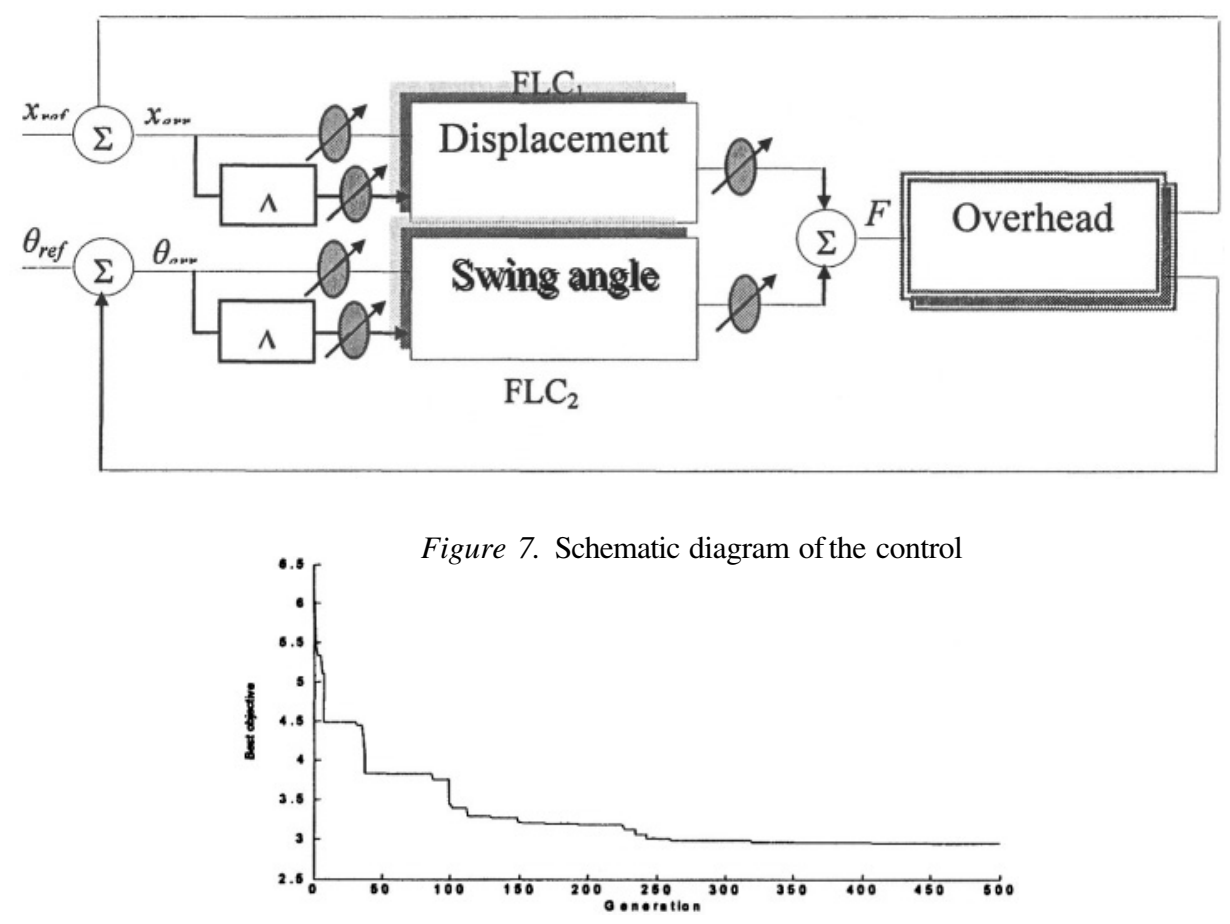

Figure 8. Evolution of the objective function.

Simulation results from initial state $(10,0,30,0)$ to target state $(10,0,0$, 0 ) are shown in figure 9. The generated force is presented by the figure 10 .

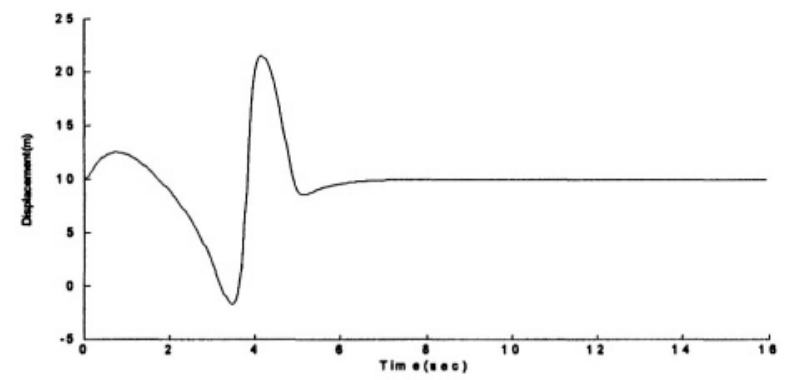

(a) 


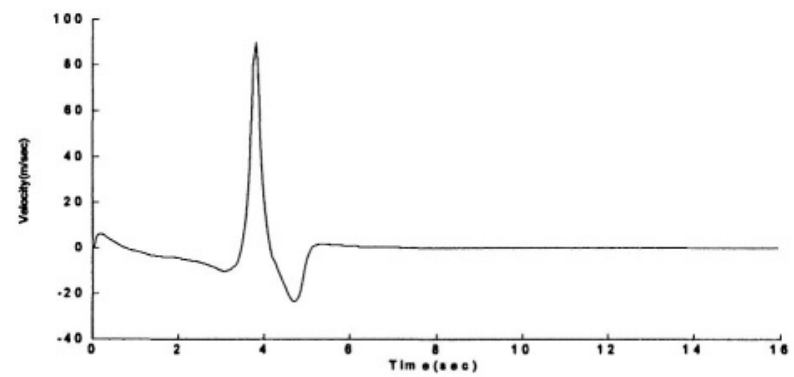

(b)

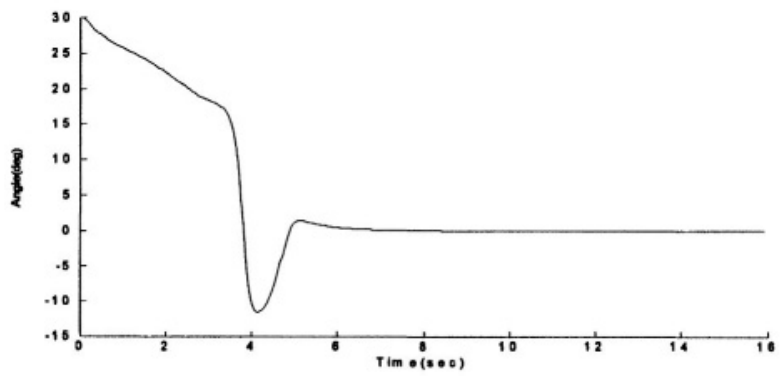

(c)



(d)

Figure 9. Evolution of the state variables.

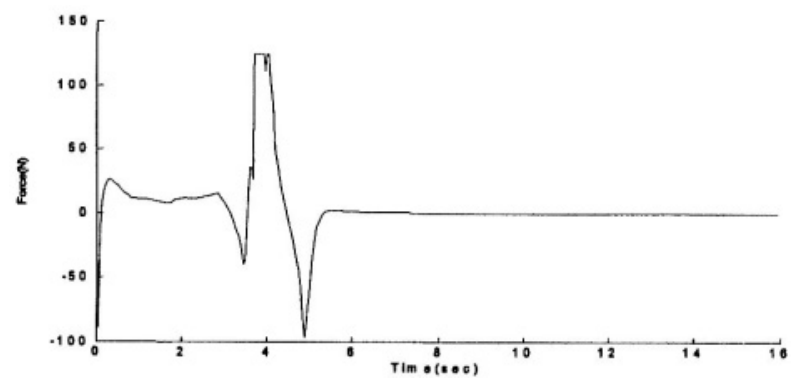

Figure 10. Output control. 


\section{CONCLUSION}

In this work, the proposed controller has been applied to control the overhead crane system. The efficiency of this approach is measured by the controller's capacity to bring back the crane to its desired state.

One can say that this controller manages to achieve the desired task, which justifies the efficiency of the proposed conception method.

The association of the fuzzy reasoning and the genetic algorithms constitute an efficient means to exploit the power and the suppleness of the elements manipulated by these different procedures and approaches intervening in the phase of the conception and add to the resulted controller an <<intelligent" functionality that is the goal aimed by the intelligent controllers.

\section{REFERENCES}

[1] L. A. Zadeh, "Fuzzy sets", Information and Control, Vol. 8, 1965, pp. 338-353.

[2] David E. Goldberg, 'Algorithmes génétiques: exploration, optimisation et apprentissage automatique', Addison-Wesley, 1994.

[3] Mamdani, E. H., "Application of fuzzy algorithms for simple dynamic plant", Proc. IEE, D-121, 1974, pp. 1558-1588.

[4] Mamdani, E. H., and Assilian, "An experiment in linguistic synthesis with a fuzzy logic controller", International Journal of Man- Machine Studies, Vol. 7, 1975, pp. 1-13.

[5] M. Sugeno and G. T. Kang, "Structure identification of fuzzy model", Fuzzy sets and systems, Vol. 28, 1988, pp.15-33.

[6] Jhy-Shing Roger Jang and Chuen-Tsai Sun, "Neuro fuzzy modeling and control", Proceedings of the IEEE, Vol. 83, No. 3, March 1995, pp. 378-406.

[7] X. W. Yan, Z. D. Deng and Z. Q. Sun, "Genetic Takagi-Sugeno-fuzzy reinforcement learning", Proceeding of the 2001 IEEE, International Symposium On Intelligent Control, September 5-7, 2001, Mexico city, Mexico, pp. 67-72.

[8] Chia-Feng Juang and Yuan-Chang Liou, "A TSK-type recurrent fuzzy network for dynamic systems processing via supervised and reinforcement learning”, 2001 IEEE International Fuzzy Systems Conference, pp. 240-243.

[9] Hao Ying, "Constructing nonlinear variable gain controllers via the Takagi-Sugeno fuzzy control”, IEEE Transactions On Fuzzy Systems, Vol. 6, No. 2, May 1998, pp. 226-234.

[10] A. Homaifar and Ed McCormick, "Simultaneous design of membership functions and rules sets for fuzzy controllers using genetic algorithms", IEEE Transactions On Fuzzy Systems, Vol. 3, No. 2, May 1995, pp. 161-176.

[11] Jinwoo Kim, Yoonkeon Moon, and Bernard P. Zeigler, "Designing fuzzy net controllers using genetic algorithms", IEEE control systems, June 1995, pp. 66-72.

[12] Jinwoo Kim, and Bernard P. Zeigler, "Designing fuzzy logic controllers using a multiresolutional search paradigm", IEEE Transactions On Fuzzy Systems, Vol. 4, No. 3, August 1996, pp. 213-226.

[13] Sanjay Kumar Sharma and George W. Irwin, "Fuzzy Coding of Genetic Algorithms", IEEE Transactions on Evolutionary Computation, Vol. 7, No. 4, August 2003, pp 344355.

[14] Liu Diantong, YI Jianqiang, TAN Min, "Proposal of GA-based two-stage fuzzy control of overhead crane”, Proceeding of IEEE TENCON'02,2002, pp. 1721-1724. 